\title{
DOCUMENT(AL)O DE IDENTIDAD: CUANDO EL DOCUMENTO NO DICE MÁS QUIÉN SOMOS, SINO LO CUENTA
}

\author{
IDENTITY DOCUMENT(ARY): WHEN THE DOCUMENT \\ NO LONGER SAYS WHO WE ARE, BUT TELLS IT
}

\author{
Alberto LOCO \\ Università LUISS - Guido Carli (Roma) \\ alberto.loco@hotmail.it
}

Resumen: Cada vez más confiamos a la red recuerdos y testimonios de nuestra vida, pero también sueños y proyecciones de como quisieramos que fuese. Entonces, los Big Data no incluyen solo la identidad de los individuos, sino también su personalidad y su estado pasional, permitiendonos, así, presentar a un individuo en los diferentes contextos en los que actúa. Por medio de Internet estamos "contando" nosotros mismos y el "documento" virtual que nos representa se extende junto a nosotros convirtiendose en "documental".

Palabras clave: Identidad. Ego. Big data.

Abstract: More and more often, we commit to the network memories and records of our life, but also dreams and projections of how we would like it to be. Therefore, the Big Data do not just include the identity of individuals, but also their personality and patemic state, allowing us, in this way, to describe one in the different contexts in which they act. Through the Internet we are "discoursivizing" ourselves and the virtual "document" that represent us is growing with us turning into a "documentary".

Key Words: Identity. Ego. Big Data. 


\section{ALBERTO LOCO}

\section{INTRODUZIONE}

II 23 giugno del 2003 la società americana Linden Lab lancia online una piattaforma informatica (per l'esattezza un MUVE, Multi-User Virtual Environment, ossia un mondo virtuale) chiamata Second Life, la cui tagline recita: your world, your immagination.

Gli utenti che si iscrivono, detti residenti, accedono allo spazio di gioco sotto forma di un avatar ${ }^{1}$ tridimensionale ideato da loro stessi e sono liberi di vivere una propria vita nei termini stabiliti dal contratto stipulato con la casa produttrice. All'interno della piattaforma è possibile viaggiare, socializzare, partecipare ad attività di gruppo e individuali, creare o scambiare beni o servizi, contribuire allo sviluppo della comunità e utilizzare una valuta virtuale convertibile in valuta reale.

Second Life è dunque un gioco di ruolo che replica molto fedelmente la vita reale (la first life), con la sostanziale differenza che permette agli utenti di essere quello che desiderano e di fare quello che vogliono senza particolari limiti.

Nel 2011 la piattaforma contava un numero di iscritti pari a 22 milioni, di cui solo 1.350.000 attivi, cifra che rappresentava all'incirca il 2,2\% della popolazione mondiale.

Va ora detto che i cambiamenti avvenuti su internet negli ultimi dieci anni, con l'affermarsi dei social network, la commercializzazione dei nuovi device mobili e l'avvio di nuove pratiche commerciali legate alla raccolta dei Big Data, hanno fornito la medesima possibilità di costruirsi un'identità alternativa a tutti i fruitori della rete. Si tratta di circa 3 miliardi di persone, la metà degli abitanti del pianeta.

Due sono gli eventi cardine verificatisi dal 2005 a oggi cui fare riferimento. II 4 dicembre 2009 un post apparso sul blog ufficiale di Google informava che ci sarebbero state "ricerche personalizzate per tutti":

Oggi Google usa 57 indicatori - dal luogo in cui siamo al browser che stiamo usando, fino al tipo di ricerche che abbiamo fatto in precedenza - per cercare di capire chi siamo e che genere di siti ci piacerebbe visitare. Anche quando non siamo online, continua a personalizzare i risultati e a mostrarci le pagine sulle quali probabilmente cliccheremo (Pariser, 2011: 9).

Nel settembre 2011 cambiava la policy di Facebook e quella che per sei anni era stata la bacheca personale di ogni utente diventava una Timeline ${ }^{2}$ ovvero, come scrive un articolista su webandmore.it:

1 Dal sanscrito incarnazione, poi esteso a indicare qualunque immagine o figura scelta per rappresentare la propria utenza su Internet.

2 FB la pubblicizzò utilizzando Andy Sparks, un adverter ovvero una persona immaginaria la cui vita viene costruita ad hoc per scopi pubblicitari. 
la registrazione cronologica di tutte le nostre azioni: gli aggiornamenti di status, le foto caricate, i like, i video condivisi, le applicazioni usate, i commenti. C'è tutto quello che abbiamo fatto da quando abbiamo aperto un profilo su Facebook. [...] Si può andare all'indietro fino alla nascita, facendo una sorta di diario a ritroso. Infatti è possibile aggiungere non solo gli aggiornamenti di stato, ma anche elementi del proprio passato: il giorno della laurea, il matrimonio, la nascita di un figlio, il giorno del divorzio e compagnia bella.

L'insieme di queste informazioni ha generato e genera un'enorme quantità di dati, Big Data appunto, che rappresentano le abitudini, la personalità, le opinioni e le relazioni di ogni utente online. Scie residue di ogni operazione fatta in internet vengono accuratamente raccolte da Google, da Facebook e da altre apparenti utilities, perché le informazioni sono una delle merci più preziose del pianeta in quanto ciò che ci lasciamo dietro ci rappresenta.

Nella dinamica di raccolta dei Big Data e dell'utilizzo dei filtri da parte delle utilities ci sono due aspetti da considerare. Uno certamente pragmatico:

In cambio del servizio che offrono i filtri, regaliamo alle grandi aziende un'enorme quantità di dati sulla nostra vita privata, molti dei quali non confideremmo neanche a un amico. [...] In conclusione la bolla dei filtri può influire sulla nostra capacità di scegliere come vogliamo vivere (Pariser, 2011: 19-20).

Un secondo aspetto riguarda invece la dimensione bulestica ${ }^{3}$ delle nostre vite:

Icreatoridella personalizzazione cioffrono un mondo sumisura, ogniaspetto del quale corrisponde perfettamente ai nostri gusti. $\dot{E}$ un mondo rassicurante, popolato dalle nostre persone, cose e idee preferite (Pariser, 2011: 16).

Pariser lascia intendere che abbiamo barattato, o forse è il caso di dire che abbiamo ceduto, i diritti dei nostri dati per la possibilità di essere qualcosa di diverso da ciò che siamo realmente. La verità scambiata con l'illusione.

In Matrix le macchine avevano preso il sopravvento sul genere umano con l'inganno, noi invece usiamo deliberatamente le macchine come oppio dei popoli.

La dinamica è la stessa di Second Life, ma non ne percepiamo la differenza. Perché?

3 Legata alla sfera del volere. 
Forse perché sembra un gioco. Postare, condividere, taggare è divertente. Eppure come risposta questa non è del tutto soddisfacente in quanto è sempre di 3 miliardi di persone online che si sta parlando.

II mio intervento non intende prendere in considerazione i risvolti etici, morali o commerciali delle nuove frontiere del marketing, ma mira a comprendere le dinamiche personali che ce ne rendono partecipi, senza che questo ci sconvolga più di tanto. Ritengo siano due i problemi che emergono dal discorso sui new media e che necessitano di essere qui esaminati: un problema di identità e un problema di ego. Occorre però fare una breve premessa.

\subsection{MONDO VIRTUALE E DATA DOUBLES}

Di mondo virtuale ne parlava già Baudrillard negli anni '90, ma è stato Bauman a porre le basi per un discorso sulla sovrapposizione di"realtà"di diversa sostanza dell'espressione. Egli considera il virtuale non come realtà parallela, ma come spazio artificiale e lo popola di entità che chiama data doubles ${ }^{4}$, cloni virtuali degli utenti costituiti dai dati che essi lasciano in rete (Bauman, 2014: XII).

Nel corso di una precedente ricerca ho definito tali cloni di dati come il risultato sincretico di una "serie di indici (connotati, coordinate spazio-temporali, opinioni, interessi, gusti, preferenze, scelte, bisogni, desideri e relazioni) scaturiti da diverse operazioni online quali accesso, registrazione, click, immissione e rimozione di contenuti, navigazione, disconnessione" (Loco, 2015). Ciò a dimostrare la complessità strutturale delle entità che popolano il mondo virtuale, ma anche la preziosità che la raccolta di questi insiemi di dati rappresenta per ambiti come il marketing, la politica e la sicurezza, che vivono di informazioni spendibili e/o riciclabili, le quali consentono di incrementare dei flussi specifici: vendite, voti, identità ecc..

È importante sottolineare che quello che è stato detto innanzi rende questi doppleganger della rete dei dataset personalizzati o, per meglio dire, dei documenti che non dimostrano solo l'identità, ma anche gli stati patemici, le abitudini e le relazioni di ogni persona clonata. Estendendo per metafora, i dataset sono classificabili come documentari riguardanti la vita di ogni utente, i quali si aggiornano di continuo senza sosta.

Questo passaggio dal documento al documentario non è immediato e soprattutto non è dovuto alla sola natura dei Big Data: essi sono un semplice strumento che consente la correlazione di più dati e dunque un processo di narrativizzazione delle matrici che li contengono. Siamo consapevoli di lasciare tracce in rete, ma restiamo incapaci di comprenderne la portata quantitativa e le conseguenze che derivano dalle analisi svolte.

4 Bauman si rifà a un termine utilizzato in una ricerca del 2000 da Kevin Haggerty e Richard Ericson. 
Siamo invece perfettamente coscienti dei risvolti qualitativi che lo stare in rete implica e questo perché siamo noi che raccontiamo la storia.

\section{COSTRUZIONE DELL'IDENTITÁ}

Tra gli innumerevoli cambiamenti che i cosiddetti nuovi media hanno apportato ce n'è uno fondamentale che da un lato modifica lo stile di fruizione, dall'altro rovescia il paradigma narrativo e introduce un diverso modo di percepire il rapporto fra sé e il mondo. Prima la televisione era l'emanazione/estensione delle vite degli altri, l'ospite invadente che entrava in casa nostra per raccontarci le sue storie, elargendo ai loro protagonisti il proverbiale quarto d'ora di celebrità. Ora invece internet è l'emanazione/ estensione del nostro ego e siamo noi che invadiamo gli altri con le nostre storie, ben consci che oggi la visibilità è a portata di un click. La differenza sta nel fatto che prima la visibilità era selettiva, adesso invece è amatoriale.

Sono esplicativi un paio di esempi: il rapper americano MattyB aveva 7 anni quando nel 2010, grazie a Youtube e con 500.000 visualizzazioni in una settimana, è diventato una celebrità alla pari di artisti del calibro di Vanilla Ice e James Maslow. Justin Bieber ha ottenuto lo stesso successo nel 2007 dopo essere stato scoperto per caso, sempre su Youtube, dall'agente artistico Scooter Braun.

Le possibilità introdotte dai new media, che consentono al consumatore di essere al contempo anche produttore di contenuti (prosumer) e che permettono di poter condividere tutto in ogni momento della propria vita (sharing), hanno dato il via a un processo di autoreferenzialità in continua evoluzione. È a questo punto che entrano in scena le logiche bulestiche del voler-essere e del voler-fare (Greimas-Courtés, 2007: 383).

La fama di una persona non la fa tanto il talento quanto l'avere un pubblico e questo vale sia nel mondo reale che in quello virtuale. Se intendo fare il cantante o il regista apro un canale Youtube e con un'appropriata promozione avrò un pubblico. Lo stesso accade se voglio divenire fotografo, scrittore, cuoco e così via, perché c'è Instagram, ci sono i blog, i video tutorial ecc.. Più cresce il numero dei miei follower, più ciò che faccio viene legittimato, finché il voler-fare non diventa voler-essere. Questo perché attribuirsi un ruolo e darsi un tono sono consequenziali.

Riprendiamo il discorso sulla Timeline: quando guardiamo quel video promozionale niente e nessuno può vietarci di credere che l'adverter di Facebook Andy Sparks esista realmente, a parte l'assurdità del fatto che abbia postato una sua foto da neonato nello stesso anno in cui è venuto al mondo. Ciò per dire che Facebook e i social in generale ci consentono di assumere un aspetto differente da quello della vita reale: chiunque può avere una vita apparentemente perfetta come quella di Andy Sparks. 
Con un po' di fantasia e alcuni post accompagnati dalle giuste foto posso dimostrare che ho scalato l'Everest, allo stesso modo di come Salgari parlava della Malesia senza essersi mai mosso da casa. Da questo punto di vista Facebook è un comodissimo strumento di menzogna per i nostri amici, quanto lo è per noi in termini di illusione. $\grave{E}$ dunque possibile costruirsi un'identità, ottenere una certa visibilità, farsi una reputazione che ci aggradi e via dicendo. In altre parole è possibile soddisfare il proprio ego.

Naturalmente non tutto deve necessariamente essere inteso come menzogna. Possono anche solo esserci semplici aspetti della nostra vita che preferiamo mettere in evidenza rispetto ad altri, che poi è quello che succede abitualmente nella maggior parte dei casi. Postare molte foto di feste o di amici può voler indicare che si ha una vita sociale molto attiva, mentre condividere opinioni di politici e articoli di giornale potrebbe voler dire che si è una persona informata, attenta a quello che succede nel mondo.

Su Facebook è più facile avere un proprio stile, usare registri linguistici inconsueti, avere differenti orientamenti, insomma cercare di essere qualcuno agli occhi di chi non ci conosce. Se guardiamo il fenomeno dal punto di vista dell'enunciazione qui non si tratta più di "ego che dice ego" (Benveniste, 2009: 112), ma di ego che dice alter ego. Si tratta di un fenomeno di automediazione, effetto collaterale di un mondo in cui tutto per esistere deve essere mediato e mediatizzato, incluse l'affermazione e la legittimazione dell'io che, nella società occidentale, passano ormai inesorabilmente attraverso il vaglio critico del popolo della rete. Vige cioè una logica dell'accettazione virtuale.

\subsection{TESTO E BIOGRAFIA}

La massima ambizione di uomo è quella di poter vivere in eterno o almeno, in alternativa, lasciare un segno del proprio passaggio che tramandi il suo nome nei secoli a venire. I social oggi consentono qualcosa di molto simile.

Sulle nostre pagine personali lasciamo delle tracce (amici, feste, relazioni, eventi), depositiamo testi (foto, canzoni, video, poesie), memorie (date, ricorrenze, anniversari), testimonianze (recensioni, localizzazioni, mappe), ragion per cui, ad esempio, la Timeline di Facebook è stata fin da subito presentata come diario. La strategia a monte non è difficile da capire perché segue un paradigma di avvicinamento: la bacheca era troppo impersonale, rimandava a un oggetto appeso in un luogo pubblico anonimo, di solito un corridoio, un atrio o una sala d'attesa, sul quale ognuno poteva lasciare dei messaggi. D'altro canto il termine diario, soprattutto per le generazioni precedenti alla mia, è legato al concetto del caro diario, sul quale si appuntavano esperienze o si esprimevano pensieri. La differenza è che ora, invece di tenerlo chiuso in un cassetto, il diario viene reso pubblico. 
Il concetto di Timeline è utile per disambiguare il processo di narrativizzazione, descritto nel paragrafo precedente, da quello di discorsivizzazione di cui parleremo ora. Infatti l'idea di linea del tempo implica una struttura sintagmatica di tipo cronologico più attinente ai fatti che agli atti. Ciò è interessante dal punto di vista enunciativo perché i due processi ibridano in parte la distinzione che Benveniste fa tra storia e discorso: noi, ogni qual volta postiamo qualcosa, diciamo io e parliamo nei tempi del presente (discorso), ma con il passare dei giorni, quell'io diventa sempre più una terza persona che si rivolge al passato (storia) perché si allontana nel tempo (Magli, 2004: 147; Manetti, 2008: 38-40).

Dunque accade che, sempre più spesso, in rete si sovrappongano il testo, che rappresenta la nostra costruzione mediatica nella stessa accezione in cui i Big Data la raccolgono, e la biografia, che è l'insieme dei fatti della nostra vita. Ciò che siamo e che facciamo si sovrappone a ciò che vogliamo-essere e vogliamo-fare.

Ora, la categoria del desiderio non appartiene propriamente alla semiotica, ma sarebbe opportuno indagare il fenomeno anche sotto l'aspetto puramente patemico. Attraverso l'assiologia desiderio/timore, e il suo corrispettivo modale introdotto da Blanché filia/fobia, si può ottenere una lettura del seguente tipo: il timore (la fuga) del mondo empirico spinge gli utenti a cercare di realizzare i propri desideri (l'avvicinamento) nel mondo virtuale. Da ciò deriva il pensiero comune che i social aiutano ad esprimersi e sono un luogo dove si può essere se stessi.

In altri termini internet ci fornisce uno spazio d'azione e interazione che probabilmente sarebbe difficile da ottenere nel mondo reale per via di tutta una serie di schemi mentali e idiosincrasie. A ciò va poi aggiunto il discorso sulla categoria timica euforia/disforia che consente di trovare un nesso tra la percezione di sé e il modo in cui si viene percepiti dagli altri (Greimas-Courtés, 2007: 79-80; Pezzini, 1998: 53-55). Ecco come lasciare un segno del proprio passaggio diventa possibile, agevolando l'affermazione del sé di cui sopra. In tal modo entriamo a far parte della storia virtuale del mondo.

Ultimamente ho constatato che su Facebook e Twitter i profili di personaggi pubblici deceduti, ma anche quelli di persone scomparse da me conosciute, sono ancora aperti e disponibili agli utenti. Particolare è il caso del profilo Twitter di Michael Jackson che è ancora operativo (gestito dalla Sony) a sei anni dalla sua scomparsa e coinvolge quasi 2 milioni di follower. Classifico questo tipo di profili come non-luoghi della memoria.

\subsection{PARADIGMA DI VICINANZA}

Va affrontato ora, nell'ambito dei new media, il discorso sul paradigma di avvicinamento o vicinanza che permette di capire in che modo si passi dalle dinamiche personali a quelle sociali. La costruzione dell'identità, come abbiamo dimostrato, è atta a 
creare effetti di realtà e di finzione applicabili a noi stessi, o meglio alle nostre proiezioni/ automediazioni, in un mondo in cui non esiste più vero o falso, ma solo veridizione.

All'interno della rete le facciate che costruiamo, quindi le pagine personali e i profili, hanno una prevalente funzione di engagement grazie alla quale si diventa parte delle sottostrutture di cui internet è composto (groups, networks, community) (Loco, 2015). Prova questa del fatto che il principale effetto pragmatico di internet è stato quello di annullare le distanze spaziali e contrarre quelle temporali. Nel momento in cui da persone diventiamo personaggi, mettiamo in atto una manovra di avvicinamento nei confronti dei contatti con i quali interagiamo, non in termini di distanze, bensì in chiave empatica e di trasparenza. La condivisione di ciò che reputiamo significativo nella nostra vita equivale a instaurare rapporti basati su gradi di confidenza sempre più estesi, pur rimanendo nel privato delle nostre case.

Dice bene Pariser che su Internet "raccontiamo cose che non diremmo a un amico" (Pariser, 2011: 20), ma è anche vero, come scrive Bauman, che la "tecnologia ci permette di operare a distanza, con distacco e automaticamente" (Bauman, 2014: 74). Una delle conseguenze collaterali dell'uso di Internet è che sta progressivamente riuscendo a privare di valore i rapporti interpersonali: quella che nel mondo virtuale prende il nome di amicizia è solo un rapporto simulato che nel mondo reale sarebbe considerato semplice conoscenza. Nonostante ciò chi fa parte della stessa cerchia online sa forse di noi più di coloro che frequentiamo abitualmente offline.

Bauman prova a spiegare questo meccanismo alla luce di una lettura antropologica scrivendo che:

ora che il vecchio incubo panottico di "non essere mai soli" ha ceduto il posto alla speranza di "non essere mai più soli" (abbandonati, ignorati e negletti, bocciati ed esclusi) la gioia di essere notati ha la meglio sull'essere svelati (Bauman, 2014: 8).

Un concetto simile è stato espresso da Baudrillard dieci anni prima, quando affermava che:

allillusione tragica del destino preferiamo l'illusione metafisica del soggetto e dell'oggetto, del vero e del falso, del bene e del male, del reale e dell'immaginario, ma, in un'ultima fase, preferiamo ancora l'illusione virtuale, quella del né vero né falso, del né bene né male, quella di un'indistinzione del reale e del referenziale, quella di una ricostruzione artificiale del mondo in cui, 
a costo di un totale disincanto, godremmo di un'immunità totale (Baudrillard, 1996: 47).

Questa riflessione non solo si ricollega al mio discorso iniziale, ma chiude il cerchio spostando l'attenzione su un tipo di fragilità umana dovuta all'epoca incerta nella quale viviamo e in cui l'identità personale perde parte della sua esclusività. Ci sono dunque due dinamiche da prendere in considerazione parlando della rete e che riguardano l'opposizione tra due coppie di categorie: aperto/chiuso e curiosità/riserbo.

La prima riguarda il soggetto dell'agire, sempre più aperto online e sempre più confidente nei confronti del suo pubblico, ma anche progressivamente più chiuso e diffidente all'esterno. Non ci sono problemi se chi mi chiede l'amicizia su Facebook viene a conoscenza di tutto ciò che mi riguarda e volendo anche di ciò che riguarda i miei amici. Invece nel momento in cui incontro una persona sicuramente passeranno mesi prima che questa meriti la mia fiducia, quindi la mia amicizia, questo perché non so che tipo è, se c'è da fidarsi o se vale la pena di approfondire la sua conoscenza.

La seconda coppia di categorie concerne invece l'atteggiamento del pubblico verso il soggetto agente: la logica dell'accettazione si muove lungo le direttrici del voler-sapere/ non-voler-far-sapere e del non-voler-sapere/voler-far-sapere. Due movimenti che vanno uno dall'interno verso l'esterno e l'altro in senso contrario. Se volessimo leggerla in chiave distopica, potremmo ricorrere al motto del romanzo The Circle di Dave Eggers:"se non sei trasparente, cosa hai da nascondere?" (Eggers, 2013).

\section{CONCLUSIONI}

Come abbiamo detto all'inizio di questo discorso i data doubles sono composti in parte da ciò che siamo e in parte da ciò che facciamo e diciamo. Allo stesso modo la nostra identità online è composta dalla nostra personalità da un lato e dal nostro ego dall'altro.

Resta da dire che gli algoritmi filtranti di Google, Facebook e simili sono programmati per attuare operazioni di sintesi: raccolgono e analizzano la nostra parte immanente, in modo da poter conoscere, talvolta prima di noi, i nostri desideri e offrirci di conseguenza delle soluzioni. $\mathrm{Ci}$ aiutano insomma a costruire la nostra parte manifesta, il nostro volto pubblico.

II processo diventa così un circolo vizioso: noi non verremo mai a conoscenza di ciò che non ci interessa o non ci piace e ci troveremo sempre più chiusi nel nostro piccolo

5 È in realtà impensabile, almeno con la consapevolezza dei nostri tempi, che Internet garantisca un'immunità totale. 
mondo ideale dove anche le persone con cui siamo in contatto non avranno idee diverse dalle nostre.

Due sono a questo punto le meccaniche da tenere in considerazione.

Una prima riguarda la caratteristica dell'uomo di inventare storie, non più sugli altri ma su se stesso e con una convinzione tale da diventare egli stesso le storie che racconta.

La seconda invece appartiene al sistema cibernetico e ha a che fare con il passaggio da ciò che era documento a ciò che è diventato documentario.

Le agenzie di raccolta dati all'inizio erano alimentate da una quantità di informazioni anagrafiche che con il tempo sono andate sempre più arricchendosi di dati su ciò che è stato fatto, detto, vissuto o, in altri termini, su ciò che un individuo era offline. Discorso che rende il dato una seconda pelle e non più unicamente un elemento informativo autonomo disperso nella rete e utile solo per sondaggi o statistiche.

II documento, che nella sua accezione più classica è "qualsiasi mezzo che provi l'esistenza di un fatto" (Vocabolario Treccani, 1987), è diventato dapprima una serie di documenti, quindi una documentazione, successivamente un documentario, ovvero una serie di documenti montati assieme che riguardano uno specifico oggetto o soggetto d'interesse. Il che spiega perfettamente tutte le pratiche autoreferenziali presenti online, dallo sharing al selfie.

Il documentario è per principio qualcosa di dinamico, di esteso, che può essere autoprodotto e costantemente aggiornato, ma soprattutto che ha la caratteristica di essere permanente. Internet ha forse la capacità ancora poco valutata di trasformare non solo il discorso in storia, ma anche la storia in memoria.

È la ragione principale per la quale è importante oggi dibattere sull'uso e sui limiti dei nuovi mezzi di comunicazione, ma anche sui problemi che essi sollevano in campi aperti quali quelli dell'etica e della deontologia. La semiotica in particolare è in grado di fornire gli strumenti adatti a elaborare nuovi modelli interpretativi che servano a riflettere su logiche di costruzione testuale in continua evoluzione, sempre più ibride e sempre più instabili. 
DOCUMENT(AL)O DE IDENTIDAD: CUANDO EL DOCUMENTO NO DICE MÁS QUIÉN

SOMOS, SINO LO CUENTA

\section{RIFERIMENTI BIBLIOGRAFICI}

BAUDRILLARD, J. (1996). I/ delitto perfetto. Milano: Raffaello Cortina Editore.

BAUMAN, Z. \& LYON, D. (2014). Sesto potere. Bari: Laterza.

BENVENISTE, É. (2009). Essere di parola. Orio Litta: Mondadori.

EGGERS, D. (2014). II cerchio. Milano: Mondadori.

GASPARATO, A. (in linea). Facebook cambia faccia: dalla bacheca alla nuova Timeline, Web and

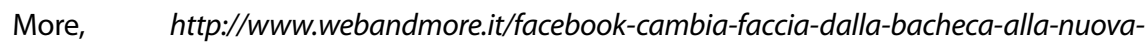
timeline/ [30/09/2015].

GREIMAS, A.J. \& COURTÉS, J. (2007). Semiotica. Dizionario ragionato della teoria del linguaggio. Milano: Mondadori.

LOCO, A. (i.p.). "Big Data: contesto, strutture, problematiche".

MAGLI, P. (2004). Semiotica. Venezia: Marsilio.

MANETTI, G. (2008). L'enunciazione. Milano: Mondadori.

PARISER, E. (2001). II filtro. Milano: il Saggiatore.

PEZZINI, I. (1998). Le passioni del lettore. Milano: Bompiani. 
